\title{
Ramas Emergentes del Arco Aórtico en Fetos Humanos. Un Estudio Descriptivo Directo en Población Colombiana
}

\author{
Branches Arise of the Aortic Arch in Human Fetus. \\ A Descriptive Direct Study in Colombian Population
}

*José Dario Rojas Oviedo \& ** Luis Ernesto Ballesteros Acuña

ROJAS, O. J. D. \& BALLESTEROS, A. L. E. Ramas emergentes del arco aórtico en fetos humanos. Un estudio descriptivo directo en población colombiana. Int. J. Morphol., 27(4):989-996, 2009.

RESUMEN: Las variaciones anatómicas en el número y ubicación de ramas emergentes del arco aórtico tienen diversos grados de implicación clínica y de su identificación oportuna depende el manejo y pronóstico de las alteraciones asociadas. Al revisar su compleja morfogénesis y reportes bibliográficos previos surge la hipótesis de si algunas formas de expresión pudieran ser transicionales durante la gestación y no manifestarse en el adulto. En Colombia no hay registros directos sobre la expresión morfológica de las ramas del arco aórtico en población fetal, razón que motiva el desarrollo del presente trabajo. Se analizaron 51 bloques cardiopulmonares de fetos mortinatos sin anomalías cardiovasculares, entre 17 y 40 semanas de gestación, cuyos lechos vasculares fueron repletados con resina poliéster. Luego se valoró el número de ramas emergentes del arco aórtico y la distancia entre ellas de acuerdo a la edad gestacional. La forma usual de presentación con 3 ramas en el arco aórtico se encontró en 56.9\% de los casos, en el 27,5\% se originaron 2 ramas, y en el 15,7\% 4 ramas. La distribución del número de ramas por edad gestacional no mostró diferencia significativa ( $>00,1)$. La distancia entre la primera y última ramas aumentó linealmente con la edad gestacional, pero no se observó diferencia significativa ( $\mathrm{p}>0,1)$ entre el número de ramas y las semanas de desarrollo de los fetos evaluados. Nuestra frecuencia de variaciones en el número de ramas del arco aórtico es superior a la reportada en la literatura, particularmente en los arcos con 4 ramas en la totalidad de los cuales el vaso adicional se ubica entre la carótida común y la subclavia izquierdas. Nuestros resultados sugieren que estos patrones morfológicos están definidos desde el período embrionario y no se modifican en el transcurso de la gestación.

PALABRAS CLAVE: Arco aórtico; Fetos humanos; Variación anatómica; Colombia.

\section{INTRODUCCIÓN}

La literatura anatómica clásica reporta la emergencia de tres vasos desde el arco de la aorta: el tronco arterial braquiocefálico, la arteria carótida común izquierda y la arteria subclavia izquierda (Moore \& Dalley, 2007; Latarjet \& Ruiz-Liard, 2005; García-Porrero \& Hurlé, 2005), de las cuales depende en su totalidad la irrigación del encéfalo. Ésta expresión morfológica se observa en la mayoría de los casos, a pesar de lo cual se ha registrado una importante variabilidad en la disposición de estos derivados arteriales que oscila entre el 5 y el 42\% (Satyapal et al., 2003; De Garis et al., 1933) y difiere según el grupo poblacional estudiado (Carvalho et al., 2005; Bathia et al., 2005).

El impacto de estas variaciones anatómicas es muy diverso ya que a pesar de pasar desapercibidos en la mayo- ría de los casos y convertirse en hallazgos incidentales de disección anatómica (Schwarzacher \& Krammer, 1989), hay algunos reportes en los cuales son diagnosticadas al evaluar patologías cardiovasculares (Akdeniz et al., 2007; Paraskevas et al., 2008) respiratorias o digestivas asociadas (Kaus et $a l, .2008$; Szpinda, 2005) e incluso en cirugía de trauma con resultados no siempre favorables (Bustamante et al., 2007; Satyapal et al.).

La morfogénesis del arco aórtico y sus ramificaciones es algo compleja y se inicia alrededor del día 29, cuando el $3^{\circ}$ y $4^{\circ}$ par de arcos embrionarios comienzan la orientación hacia el patrón vascular definitivo (Moore \& Persaud, 1999), dando origen el tercer par de vasos primitivos a las arterias carótidas comunes e internas, a través de sus seg-

\footnotetext{
* Médico y Cirujano. Candidato a Magister en Ciencias Básicas Biomédicas.Profesor Asistente Universidad Industrial de Santander, Bucaramanga, Colombia

** Médico y Cirujano. Magister en Morfología. Profesor Titular Universidad Industrial de Santander, Bucaramanga, Colombia.
} 
mentos proximal y distal, respectivamente. Por su parte, el cuarto arco izquierdo participa en la configuración de la porción arqueada de la aorta con el aporte proximal del saco aórtico y distal de la aorta dorsal, mientras que del lado derecho da origen a la porción proximal de la arteria subclavia, la cual se complementa en su porción distal por la $7^{\mathrm{a}}$ arteria intersegmentaria dorsal del mismo lado, en tanto que la subclavia izquierda se origina en su totalidad de la $7^{\mathrm{a}}$ arteria intersegmentaria dorsal izquierda, consolidando así la disposición semejante a la arterial adulta entre la sexta y la octava semanas de desarrollo (Sadler, 2004).

La frecuencia de variabilidad en estos vasos sanguíneos ha sido ampliamente estudiada y múltiples análisis se han realizado desde la perspectiva de la morfología del desarrollo, de los cuales nos llaman particularmente la atención aquellos relacionados con los arcos de dos ramas bien por presencia de origen común bicarotideo o por presencia del tronco braquiocefálico izquierdo (Zamir \& Sinclair, 1990; Grande et al., 1995), aunque dichos análisis han sido realizados en muestras obtenidas de población adulta y no en población fetal, pues si bien existen estudios morfométricos ecográficos y directos sobre los grandes vasos arteriales en fetos (Noomcharoen \& Uerpairojkit, 2008; Halley Castillo et al., 2005; Szpinda et al., 2006), no hay en ellos referencias sobre tal variabilidad y salvo reportes de hallazgos específicos (Chaoui et al., 2008), el único estudio encontrado en población fetal sobre el tema, registra la presencia de tronco braquiobicarotideo, sin que se mencionen otros hallazgos sobre variabilidad (Szpinda, 2007).

En población colombiana la única descripción del número de ramas emergentes del arco de la aorta, consistió en un estudio directo realizado en nuestra Universidad, con una muestra relativamente pequeña de piezas anatómicas adultas (Herrera et al., 2007), pero ningún trabajo ha sido desarrollado en fetos humanos.

El objeto del presente trabajo es determinar las diferentes formas de expresión morfológica macroscópica de los derivados vasculares de los arcos embrionarios aórticos III y IV en una muestra de fetos humanos y analizar la hipótesis de si algunas formas observadas en adultos podrían corresponder a persistencia de formas fetales transitorias que normalmente se modifican durante el curso de la gestación.

\section{MATERIAL Y MÉTODO}

Previo consentimiento informado, se obtuvieron 51 bloques cardiopulmonares de fetos productos de abortos espontáneos o mortinatos entre 17 y 40 semanas de gestación, mediante autopsia médico científica practicada en la morgue del Departamento de Patología de la Universidad Industrial de Santander a productos de abortos espontáneos y mortinatos del Hospital Universitario de Santander.

La edad gestacional de los fetos evaluados fue calculada por biometría fetal, tomando como principal parámetro la longitud del pie y en segunda instancia, la correlación entre peso y talla para la edad gestacional a partir de las tablas adoptadas por el Departamento de Patología (García et al., 2008). Se excluyeron los productos con anomalías externas, cardiopatías o en mal estado de preservación.

Estos bloques fueron fijados durante no más de 24 horas en formaldehído al $10 \%$ y luego repletados con una mezcla previamente estandarizada en los Laboratorios del Departamento de Ciencias Básicas de la Universidad Industrial de Santander para la realización de un estudios sobre vasos sanguíneos cerebrales (Ayala et al., 2007), la cual contenía resina poliéster insaturada de viscosidad media Poliser P-2100®, estireno al 20\% como solvente, $0,1 \%$ de cobalto acelerante y $1 \%$ de peróxido de metiletilcetona (MEK) como catalizador de la reacción de fraguado, a la cual se agregó colorante rojo mineral. Todos estos productos se obtuvieron de la casa comercial Suministros Industriales SUIN S.A., Colombia.

Posterior al fraguado de la resina a temperatura ambiente fueron colocados nuevamente en la solución de formaldehído al $10 \%$ y en un tiempo no superior a las 72 horas se dio inicio al proceso de disección de las muestras, el cual requirió de instrumental de microcirugía y equipo de magnificación visual, quedando finalmente el corazón con sus elementos vasculares tributarios y emergentes. A continuación se procedió a identificar el número de vasos sanguíneos emergentes del arco aórtico, describiendo su configuración y localización con su respectivo registro fotográfico digital de alta resolución.

Para el análisis estadístico que incluyó medidas de tendencia central y análisis de regresión, se agruparon las muestras en intervalos de 4 semanas correspondientes a los meses $5^{\circ}$ a $10^{\circ}$ de gestación. Se empleó el paquete estadístico STATA 8.0

\section{RESULTADOS}

De los 51 fetos evaluados $28(55 \%)$ eran de género masculino y $23(45 \%)$ de género femenino. El número y la edad gestacional de los fetos evaluados según su género se detallan en la Tabla I. 

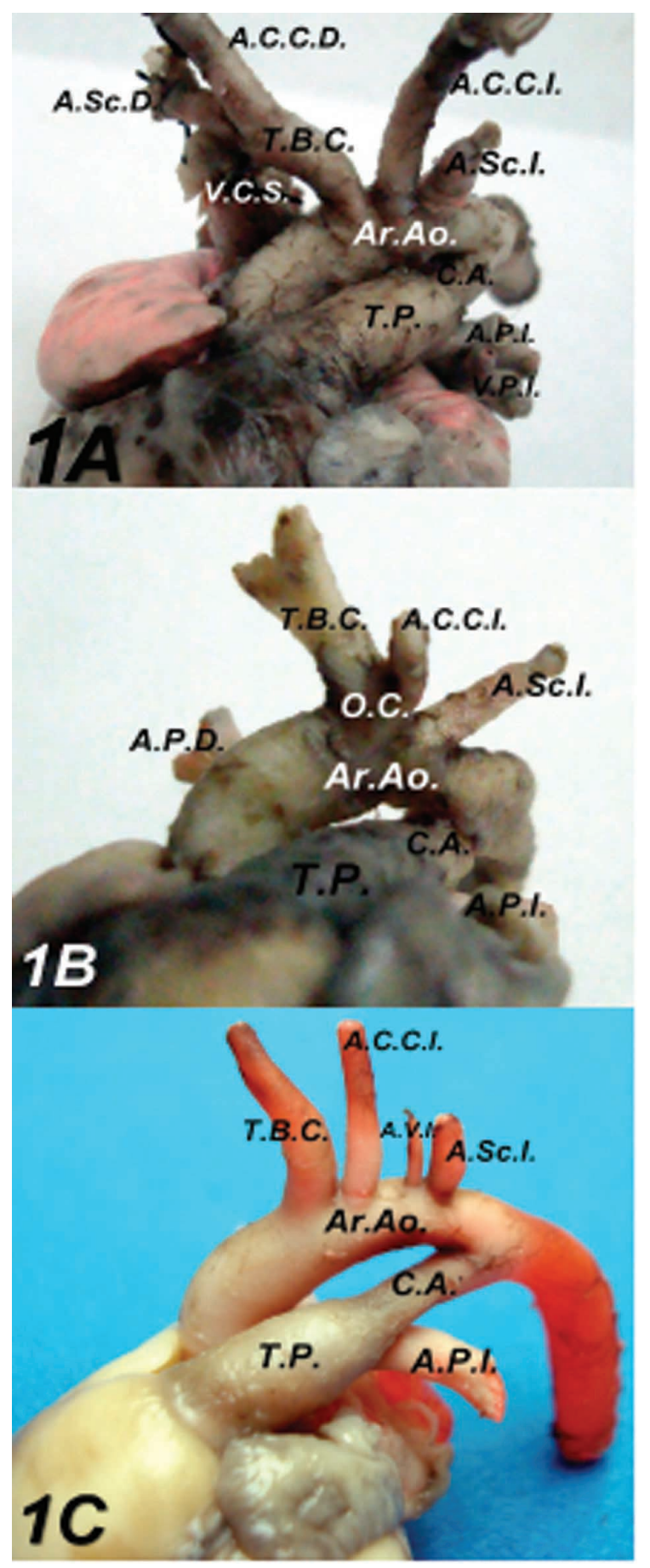

Fig. 1. Variaciones en el número de ramas emergentes del arco aórtico. $1 \mathrm{~A}$ : Arco con 3 ramas. $1 \mathrm{~B}$ : Arco con 2 ramas. 1 C: Arco con 4 ramas. Ar.Ao.: Arco aórtico, T.B.C.: Tronco Braquiocefálico, A.C.C.D.: Arteria carótida común derecha, A.Sc.D.: Arteria subclavia derecha, A.C.C.I.: Arteria carótida común izquierda, A.Sc.I.: Arteria subclavia izquierda, A.V.I.:Arteria vertebral izquierda, V.C.S.: Vena cava superior, T.P.: Tronco pulmonar, C.A.: Conducto Arterioso, A.P.I.: Arteria pulmonar izquierda, V.P.I.: Venas pulmonares Izquierdas, O.C.: Origen común de TBC y ACCI
Tabla I. Edad gestacional y género de los fetos examinados.

\begin{tabular}{lccc}
\hline $\begin{array}{l}\text { Edad gest acional } \\
\text { (semanas) }\end{array}$ & $\mathbf{n}$ & Masculino & Femenino \\
\hline $17-20$ & 5 & 2 & 3 \\
$21-24$ & 16 & 11 & 5 \\
$25-28$ & 12 & 5 & 7 \\
$29-32$ & 7 & 2 & 5 \\
$33-36$ & 4 & 3 & 1 \\
$37-40$ & 7 & 5 & 2 \\
\hline TOTAL & $\mathbf{5 1}$ & $\mathbf{2 8}$ & $\mathbf{2 3}$ \\
\hline
\end{tabular}

Se encontraron 3 formas de expresión morfológica en lo concerniente al número de ramificaciones del arco aórtico (Fig. 1). La forma usual descrita en la literatura con tres ramas fue el más frecuente en todos los grupos etáreos con 29 casos $(56,9 \%)$, seguido de la presencia de dos ramificaciones con 14 casos $(27,5 \%)$ y finalmente la presencia de 4 ramas fue la menos frecuente con 8 casos $(15,7 \%)$. No se encontró diferencia significativa entre el número de ramas y el género de los fetos evalua$\operatorname{dos}(\mathrm{p}>0,1)$.

El grupo caracterizado por dos ramificaciones, a su vez se subdivide en dos formas de presentación: una condición en la cual desde una base amplia tienen origen común el tronco arterial braquiocefálico derecho y la arteria carótida común izquierda; situación que se presentó en 12 de nuestros registros $(23,5 \%)$ y otra denominada tronco braquiobicarotideo en la cual desde un pedículo común se originan los dos vasos antes mencionados, observada en 2 casos, $(3,9 \%$ del total de muestras examinadas) (Fig. 2). Es importante anotar que en algunos ca-

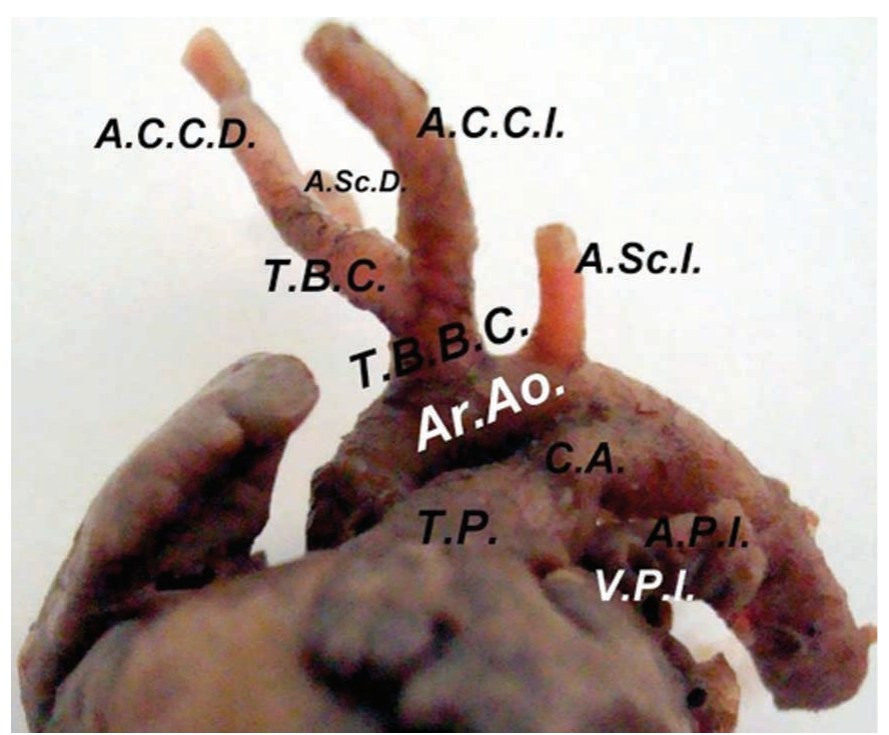

Fig. 2. Tronco braquiobicarotideo. (T.B.B.C.). Se observa la presencia de un pedículo común del cual derivan el tronco braquiocefálico (T.B.C.) y la arteria carótida común izquierda (A.C.C.I.) 


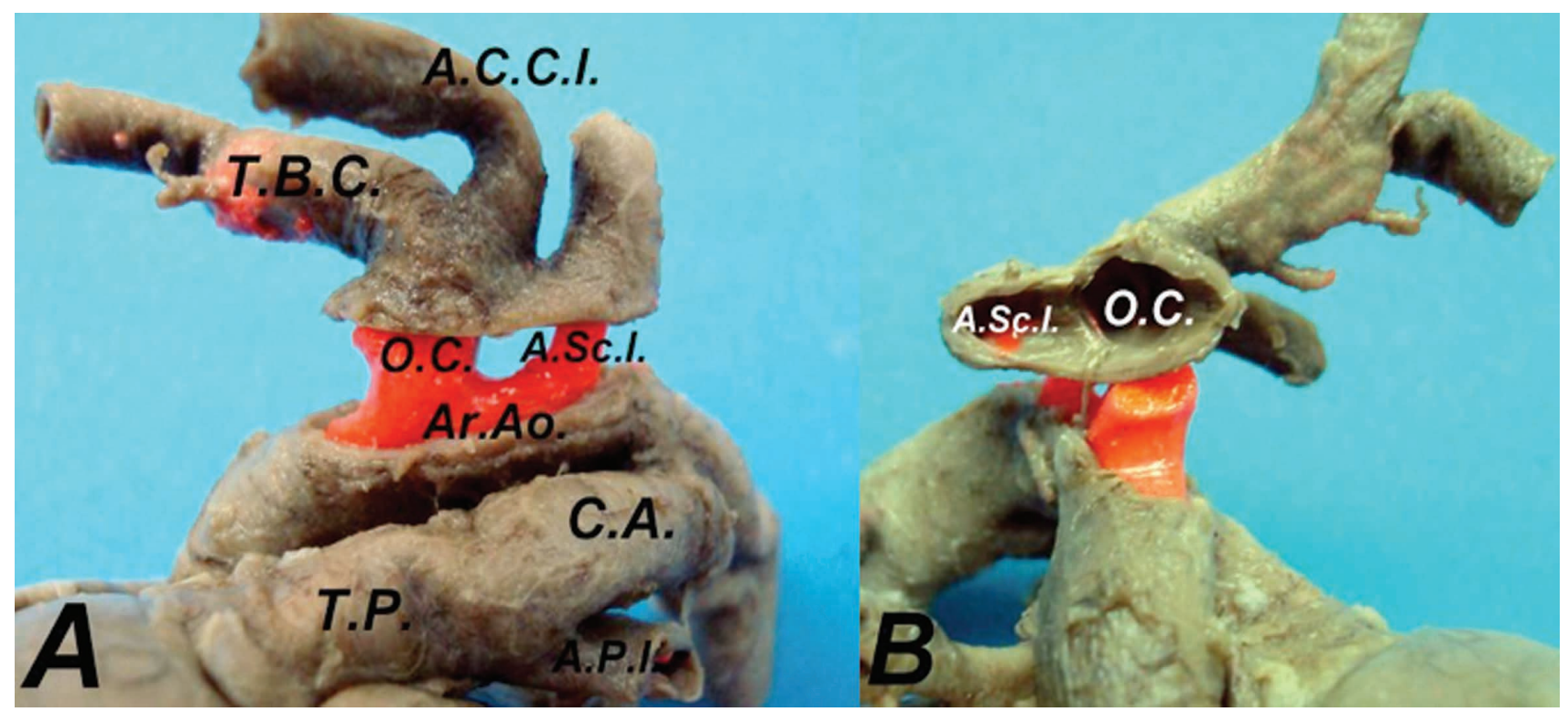

Fig. 3. Origen común del tronco braquiocefálico y la arteria carótida común izquierda. A. Evidencia de un solo pedículo en resina para los 2 vasos. B. Orificio único del cual se desprenden el tronco braquiocefálico (T.B.C) y la arteria carótida común izquierda (A.C.C.I.).

sos donde el origen común parecía algo dudoso y existía la posibilidad de encontrar dos orificios muy cercanos y no uno solo, se realizó un corte del tejido vascular confirmando que solo había un pedículo y su respectivo ostium desde la porción convexa del arco aórtico (Fig. 3).

El número de ramas por grupo de edad gestacional y sus porcentajes se presenta en la Tabla II e igualmente se representa de manera gráfica su distribución y comportamiento durante el lapso evaluado (Fig. 4).
$\mathrm{Al}$ analizar la distancia entre la primera y última rama del arco aórtico en relación con el número de colaterales que se originan de éste segmento vascular, se observa que esta aumenta progresivamente con la edad gestacional. Para los arcos con dos ramas su longitud media fue de $6,23 \mathrm{~mm}$. con DE 2,71, los de tres ramas registraron una media de $7,37 \mathrm{~mm}$. y DE 2,17, mientras que la distancia en los arcos de cuatro ramas midió en promedio $8,4 \mathrm{~mm}$. con DE 2,83, sin que se encontrara diferencia significativa entre estas mediciones $(\mathrm{p}=0,12)$ (Fig. 5).

\section{NUMERO DE RAMAS POR EDAD GESTACIONAL}

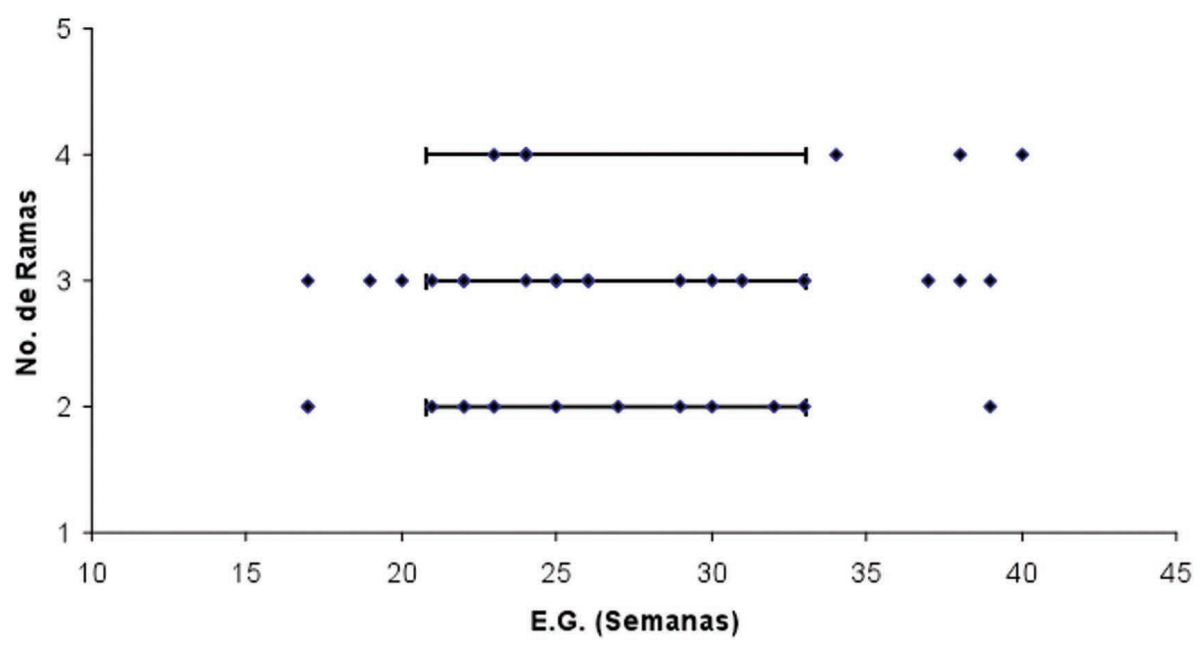

Fig. 4. Número de ramas del arco aórtico según la edad gestacional de los fetos evaluados. 


\section{DISTANCIA ENTRE RAMAS DEL ARCO AORTICO}

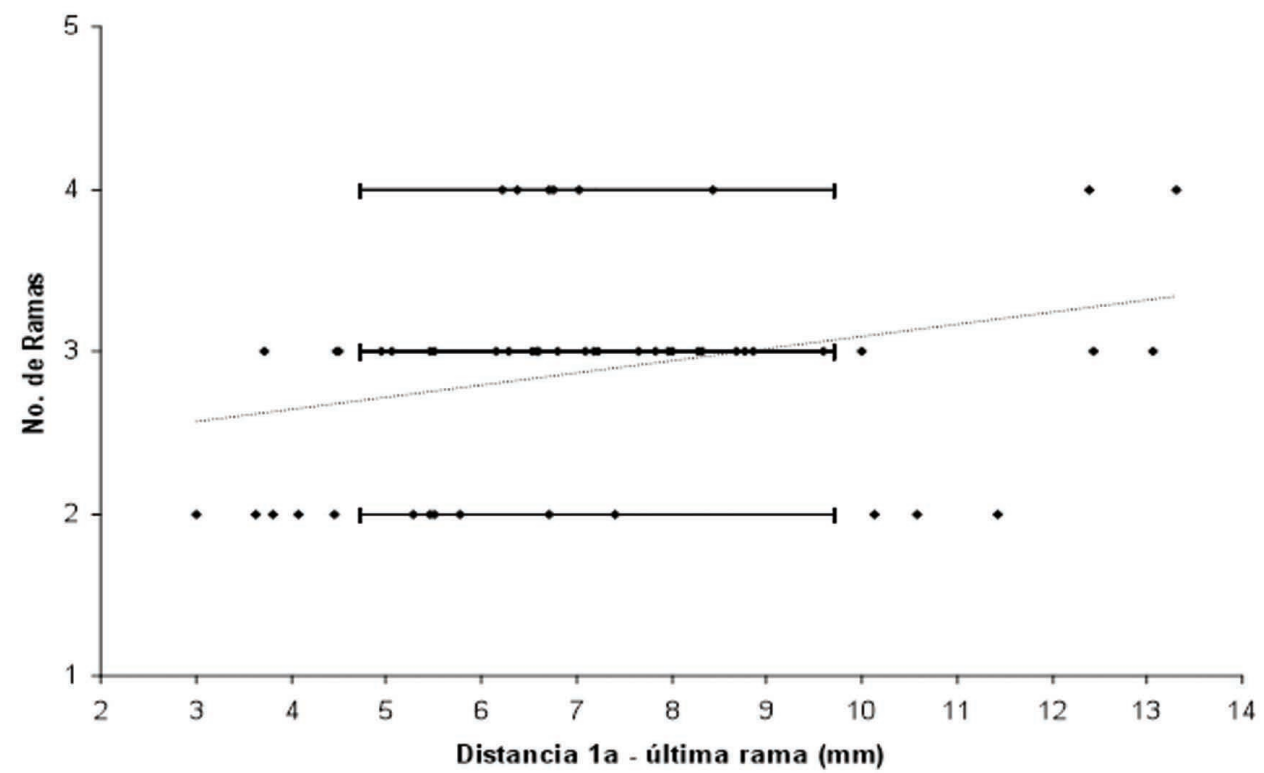

Fig. 5. Relación de la distancia entre la $1^{\mathrm{a}} \mathrm{y}$ última rama del arco aórtico según el número de ramas.
Tabla II. Número de ramas del arco aórtico por edad gestacional.

\begin{tabular}{lrrrrrr}
\hline \multirow{2}{*}{$\begin{array}{l}\text { Edad gestacional } \\
\text { (semanas) }\end{array}$} & \multicolumn{2}{c}{$\mathbf{2}$ ramas } & \multicolumn{2}{c}{$\mathbf{3}$ ramas } & \multicolumn{2}{c}{ 4 ramas } \\
\cline { 2 - 7 } & $\mathbf{n}$ & $\mathbf{\%}$ & $\mathbf{n}$ & $\mathbf{\%}$ & $\mathbf{n}$ & $\mathbf{\%}$ \\
\hline $17-20$ & 2 & 40,0 & 3 & 60,0 & - & - \\
$21-24$ & 5 & 31,3 & 6 & 37,5 & 5 & 31,3 \\
$25-28$ & 2 & 16,7 & 10 & 83,3 & - & - \\
$29-32$ & 3 & 42,9 & 4 & 57,1 & - & - \\
$33-36$ & 1 & 25,0 & 2 & 50,0 & 1 & 25,0 \\
$37-40$ & 1 & 14,3 & 4 & 57,1 & 2 & 28,6 \\
\hline TOTAL & $\mathbf{1 4}$ & $\mathbf{2 7 , 5}$ & $\mathbf{2 9}$ & $\mathbf{5 6 , 9}$ & $\mathbf{8}$ & $\mathbf{1 5 , 7}$ \\
\hline
\end{tabular}

\section{DISCUSIÓN}

Analizar las diferencias en el número de ramas derivadas del arco aórtico en población fetal es muy importante, teniendo en cuenta que la morfología del desarrollo es el fundamento de la expresión fenotípica definitiva, además de sus múltiples implicaciones clínicas y quirúrgicas. Algunos estudios morfométricos previamente citados no incluyen datos sobre éste aspecto, haciendo suponer en todos ellos la presencia de 3 vasos, sin que sea claro si por exclusión de patrones diferentes a éste o porque toda su casuística registró este número de ramas (Halley Castillo et al.; Szpinda et al.). Como dato llamativo se encuentra en la literatura un registro de fetos a término de rata, en el cual se encontró un $98,1 \%$ de arcos con 3 ramas y sólo un caso entre los 53 evaluados con 4 ramas (Monnerau et al., 2005).
En el presente estudio predomina el arco de 3 ramas en todas las edades gestacionales, aunque la variabilidad global es superior al único registro local que mostró un índice de variación cercano al 18\% (Herrera \& Ballesteros) y en general a la citada en casi toda la literatura publicada, cuyos datos han sido obtenidos de diversas formas; bien sea en población infantil y adulta por estudios angiográficos con registros variables del número de formas de expresión (Natsis et al., 2009), donde se describen 8 patrones diferente y una tasa de variación del $17 \%$, o por estudios con cadáveres (Gupta \& Sodhi, 2005; Nayak et al., 2006) donde se describen $33 \%$ de muestras con número de ramas diferente de 3 , siendo estas dos cifras concordantes con los datos citados en nuestros textos de anatomía con un máximo de variabilidad del $35 \%$.

El porcentaje de nuestros casos en los cuales el arco aórtico generó un número de ramas mayor o menor que 3 fue de 43,1, valor superior incluso a un registro bastante antiguo (De Garis et al.) no citado en los mencionados textos, correspondiente al $41,7 \%$ de variabilidad en una serie de 314 cadáveres de adultos y niños, blancos y negros de población norteamericana, en los cuales se describieron hasta 15 
patrones diferentes y en el cual si se analiza de manera aislada la población de etnia negra se encuentra que solamente un $47,7 \%$ de dichos arcos presentan la forma considerada usual en la población.

El interés de evaluar el número de ramas emergentes del arco aórtico en población fetal se fundamenta en algunas hipótesis planteada años atrás sobre la posible fusión de algunos vasos sanguíneos (Grande et al.) y en los resultados de un trabajo adelantado en Canadá (Zamir \& Sinclair), quienes formularon la teoría de que estas diferencias son el resultado de variaciones en el curso del desarrollo embrionario.

A partir de éstos planteamientos se consideró como una posibilidad el hecho de que los arcos con solo dos ramas consistieran efectivamente en un evento transicional y que en la medida en que el arco se elonga con el avance de la gestación lo que podría ser un aparente origen común del tronco braquiocefálico y la carótida común izquierda se separaran y se obtuviera un número final de 3 ramas. El análisis del comportamiento del número de ramas de acuerdo a la edad gestacional no demostró diferencia significativa entre estos dos parámetros, es decir, hay arcos de 2 ramas distribuidos equitativamente a lo largo de toda la gestación y no concentrados al inicio y disminuidos o ausentes al final, lo cual permite desmentir esta hipótesis y afirmar que la presencia del número de ramas del arco aórtico es una condición que está definida desde el inicio de su configuración y se mantiene inmodificable durante toda la gestación.

En relación con el género de estos fetos, se destaca que Grande et al. reportan en su estudio que los arcos con dos ramas todos pertenecían a fetos femeninos, en tanto que los de 4 ramas correspondían a individuos masculinos, situación no registrada en otros trabajos y que tampoco se observó en el actual, pues tanto en los arcos de 2 como en los de 4 ramas la relación de género fue exactamente 1:1.

Uno de los hallazgos más relevante es el que involucra un único origen para los vasos carotideos comunes por su implicación en la irrigación encefálica y cuya presencia en la forma de 2 ramas en el 27,5\% de los casos aquí evaluados es similar a otros reportes que oscilan entre el 3 y $30 \%$ (Satyapal et al.). Esta condición ha sido postulada como factor de riesgo para compromiso neurológico por posible déficit de irrigación, aunque al evaluarla en dos grupos de neonatos que requirieron circulación extracorpórea, uno de 20 niños con este hallazgo y otro de 111, en cuyos arcos se originaban de forma independiente las 2 arterias carótidas comunes, no se encontró significancia estadística como factor de riesgo para secuelas neurológicas (Lamers et al., 2004), siendo importante anotar que no hay estudios posteriores similares que ratifiquen o no estos hallazgos.
Dentro del grupo de arcos aórticos con una única fuente de irrigación carotídea, la frecuencia de tronco braquiobicarotídeo encontrada en esta investigación de 3,9\% es inferior al único reporte de la literatura en muestras fetales directas realizado por Szpinda (2007), quien registró esta presencia en el $21,1 \%$ de los 128 fetos examinados y también menor al $8 \%$ encontrado en una pequeña casuística en población adulta coreana (Shin et al., 2008), mientras que un estudio angiográfico realizado en Suráfrica reveló una frecuencia de tan solo el 1.6\% para esta expresión morfológica (Satyapal et al.). Sin embargo nuestra frecuencia se ubica dentro del amplio rango señalado en la literatura que oscila entre $0,47 \%$ y el $28,2 \%$ (Szpinda, 2007), aunque debe mencionarse que no todos los estudios sobre el tema reportan explícitamente la presencia de un pedículo común para los dos vasos carotídeos, quedando la duda si en aquellos reportes de alta prevalencia de esta variante podría tratarse de lo que ha sido descrito como el origen común del tronco braquiocefálico y la carótida izquierda.

Acerca de los arcos de 4 ramas cuya expresión también es diversa, un estudio reciente describe tres patrones: el primero y más comúnmente reportado en el cual la arteria vertebral izquierda emerge entre la carótida común y subclavia izquierdas; el segundo en el cual la tiroidea ima emerge entre el tronco braquiocefálico y la carótida común izquierda y el tercero, en el que un ramo mediastinal ocupa el lugar de la tiroidea ima (Gupta \& Sodhi). No incluye su registro una cuarta forma descrita previamente en la cual no hay tronco braquiocefálico derecho y la subclavia y carótida común de ese lado se originan de forma independiente (Wright, 1969), ni otra extraña condición registrada, en la cual el vaso adicional corresponde a la arteria vertebral derecha como última rama del arco (Higashi, 2008).

En nuestra casuística todos los registros correspondieron a una rama adicional entre la carótida común y la subclavia izquierda y aunque no siempre fue posible establecer su trayectoria completa, en la mayoría de los casos parece corresponder a la arteria vertebral y su frecuencia de $15,7 \%$ es superior a cualquier otro reporte previo en la literatura, incluso en aquellos estudios en los que se presentan varias formas de presentación, con $11 \%$ en un grupo de 100 cadáveres adultos (Gupta \& Sodhi).

A pesar de la tendencia creciente en la distancia entre la primera y última ramas del arco acorde a la edad gestacional no se encontró significancia estadística entre los arcos con 2, 3 o 4 ramas, esto tal vez, en relación con el tamaño muestral, lo cual genera la necesidad de determinar no sólo éste hallazgo sino los anteriores en otros grupos etáreos, aportando elementos de juicio que orienten el proceder diagnóstico y terapéutico ante ésta diversidad. 
AGRADECIMIENTOS: A todo el personal del Departamento de Patología de la Universidad Industrial de Santander, por su colaboración en el proceso de obtención de las piezas anatómicas.

ROJAS, O. J. D. \& BALLESTEROS, A. L. E. Branches arise of the aortic arch in human fetus. A descriptive direct study in Colombian population. Int. J. Morphol., 27(4):989-996, 2009.

SUMMARY: The anatomic variations in position and number of branches arise of the aortic arch have different grades of clinical implication and the right treatment and prognosis of associated anomalies depend of their early diagnosis. Reviewing your morphogenesis complex and previous literature come up the hypothesis about some expression could be transitional during gestation and do not to express in postnatal life. The morphological expression of the branches of the aortic arch in human fetuses is unknown in Colombia and that is why this study has been developed. 51 cardiopulmonary blocks obtained from stillborn fetus from 17th to 40th gestation weeks without cardiovascular anomalies, were analyzed and filled with polyester resin. Then the number of branches arises from the aortic arch and the distance between them was also analyzed through gestation. In $56.9 \%$ of the cases 3 branches are arisen from the aortic arch, 2 branches in $27.5 \%$ and 4 branches in $15.7 \%$. The number of branches according to gestational age showed no significant difference $(\mathrm{p}>0.1)$. Distance between the first and the last branches increases linearly with gestational age, but showed no significant difference ( $p>0.1$ ) between the number of branches and the gestation's weeks of the analyzed fetus. The variations found in the number of branches from the aortic arch are further than what literature reports, especially in cases with 4 branches, in these cases the additional branch was between left common carotid and left subclavian artery. These findings indicate than the morphologic patterns were defined since the embryonic stage, and they do not change during gestation.

KEY WORDS: Aortic arch; Human fetus; Anatomic variations; Colombia.

\section{REFERENCIAS BIBLIOGRAFICAS}

Akdeniz, B.; Yilmaz, E.; Pekel, N. \& Ergul, B. Anomalous origin of the right vertebral artery from the ascending aorta in the presence of an aberrant right subclavian artery. Int. J. Cardiovasc. Imaging, 23:39-42, 2007.

Ayala, J.; Ballesteros, L.; Suárez, D. \& Forero, P. Estandarización de una técnica de inyección de resina poliéster para el estudio de la vasculatura cerebral. Revista Salud UIS, 39:169-74, 2007.

Bathia, K.; Ghabriel, M. N. \& Henneberg, M. anatomical variations in the branches of the human aortic arch: a recent study of a South Australian population. Folia Morphol. (Warsz), 64(3):217-23, 2005.

Bustamante, N.; Galletti, C.; Marangoni, A.; Bronzi, J.; Londero, H. \& Delfino, A. Traumatismo cerrado del tronco arterial innominado, variedad anatómica tipo bovino. Tratamiento de un caso y revisión de la literature. Rev. Fed. Arg. Cardiol., 36:94-8, 2007.

Carvalho, M.; Araújo, J.; Gomes, L. \& Panerai, C. Prevalência de variações anatômicas do arco aórtico em um grupo de indivíduos brasileiros. Int. J. Morphol., 23(1):45-94, 2005.

Chaoui, R.; Rake, A. \& Heling, K. Picture or the month. Aortic arch with four vessels: aberrant right subclavian artery. Ultrasound Obstet. Gynecol., 31:115-7, 2008.
De Garis, C.; Black, I. \& Riemenschneider, E. Patterns of the aortic arch in American White and Negro stocks, with comparative notes on certain other mammals. J. Anat., LXVII(4):599-619, 1933.

Garcia, J.; Monroy, S. \& Garcia, C. Principios de procesamiento en patología quirurgica general y por sistemas. Colombia, Universidad Industrial de Santander, 2008. p.378.

García-Porrero, J. \& Hurlé, J. Anatomía humana. Madrid, McGraw- Hill Interamericana, 2005. pp.579-712.

Grande, N.; Costa, A.; Silva, E.; Sousa, A. \& Aguas, A. Variations in the anatomical organization of the human aortic arch. A study in a Portuguese population. Bull. Assoc. Anat. (Nancy), 79(244):19-22, 1995.

Gupta, M. \& Sodhi, L. Variations in branching pattern, shape, size and relative distances of arteries arising fron arch of aorta. Nepal. Med. Coll. J., 7(1):13-7, 2005.

Halley Castillo, E.; Arteaga-Martinez, M.; Garcia, I.; VillasisKeever, M.; Aguirre, O.; Moran V, et al. Morphometric Study of the Human Fetal Heart. Clin. Anat., 18:260-8, 2005.

Herrera, N.; Ballesteros, L. \& Mantilla, J. Expresión morfológica del arco aórtico y sus colaterales. Estudio 
anatómico directo. Memorias del III Congreso Nacional de Morfología. MedUnab, 10(3):231, 2007.

Higashi, N.; Shimada, H.; Simamura, E. \& Hatta, T. Right vertebral artery as the fourth branch of the aortic arch. Anat Sci Int., 83(4):314-8, 2008.

Kaus, T.; Gasser, J.; Lesnik, G.; Sinzig, M. \& Hausegger, K. Images in vascular medicine. Two exceptionally rare branching patterns of the aortic arch. Vasc. Med., 13:8990, 2008.

Lamers, L.; Rowland, D.; Seguin, J.; Rosenberg, E. \& Reber, $\mathrm{K}$. The effect of common origin of the carotid arteries in neurologic outcome after neonatal ECMO. J. Pediatr. Surg., 39:532-6, 2004.

Latarjet, M. \& Ruiz-Liard, A. Anatomía Humana. 4 ed. Madrid, Editorial Médica Panamericana, 2005. pp.9671023.

Moore, K. \& Dalley, A. Anatomía con orientación clínica. 5a Ed. Buenos Aires, Editorial Médica Panamericana, 2007. pp.141-75.

Moore, K. \& Persaud, T. Embriología Clínica. 6 ${ }^{\mathrm{a}}$ Ed. México DF., McGraw-Hill Interamericana, 1999. pp.370-425

Monnereau, L.; Carretero, A.; Berges, S.; Navarro, M.; Leonard, M.; Lyazrhi, F.; Sautet, J. \& Ruberte, J. Morphometric study of the aortic arch and its major branches in rat fetuses on the $21^{\text {st }}$ day of gestation. Anat. Embryol. (Berl), 209(5):357-69, 2005.

Natsis, K.; Tsitouridis, I.; Didagelos, M.; Fillipidis, S.; Vlasis, K. \& Tsikaras, P. Anatomical variations in the branches of the human aortic arch in 633 angiographies: clinical significance and literature review. Surg. Radiol. Anat., 31(5):319-23, 2009.

Nayak, S.; Pai, M.; Prabhu, L.; D’Costa, S. \& Shetty, P. Anatomical organization of aortic arch variations in the India: embryological basis and review. J. Vasc. Bras., 5(2):95-100, 2006.

Noomcharoen, O. \& Uerpairojkit, B. Population-related differences in fetal arotic arch dimensions. Int. J. Gynaecol. Obstet., 102(1):72-3, 2008.

Paraskevas, G.; Agios, P.; Stavrakas, M.; Stoltidou, A. \& Tzaveas, A. Left common carotid artery arising from the brachiocephalic trunk: a case report. Cases J., 1(1):83, 2008.
Sadler, T. Langman Embriología médica con orientación clínica. $9^{\mathrm{a}}$ Ed. Buenos Aires, Editorial Médica Panamericana, 2004. pp.233-87.

Satyapal, K.; Singaram, S.; Partab, P.; Kalideen, J. \& Robbs, J. Aortic arch branch variations - case report and arteriographic analysis. S. Afr. J. Sur, 41(2):48-50, 2003.

Schwarzacher, S. \& Krammer, E. Complex anomalies of the human aortic arch system: Unique case of both vertebral arteries as additional branches of the aortic arch. Anat. Rec., 225:246-50, 1989.

Shin, I.; Chung, Y.; Shin, W.; Im, S.; Hwang, S. \& Kim, B. A Morphometric Study on Cadaveric Aortic Arch and Its Major Branches in 25 Korean Adults : The Perspective of Endovascular Surgery. J. Korean Neurosurg. Soc., 44:78-83, 2008.

Szpinda, M. A new variant of aberrant left brachiocephalic trunk in mam: case report and literature review. Folia Morphol. (Warsz), 64(1):47-50, 2005.

Szpinda, M.; Brazis, P.; Elminowska-Wenda, G. \& Wisniewski, M. Morphometric study of the aortic and great pulmonary arterial pathways in human foetuses. Ann. Anat., 188:25-31, 2006.

Szpinda, M. Morphometric study of the brachiobicarotid trunk in human fetuses. Ann. Anat., 189:569-74, 2007.

Wright, N. Dissection study and mensuration of the human aortic arch. J. Anat., 104(2):377-385, 1969.

Zamir, M. \& Sinclair, P. Continuum análisis or común branching patterns in the human arch of the aorta. Anat. Embryol., 181:31-6, 1990.

Direccion para correspondencia:

Rojas Oviedo José Darío

Carrera 32 No. $29-31$.

Departamento de Ciencias Básicas

Facultad de Salud.

Bucaramanga

COLOMBIA

Email: darojaso@gmail.com

Recibido : 04-05-2009

Aceptado: 27-08-2009 